\title{
Smooth Muscle Precursor Cells Derived from Human Pluripotent Stem Cells for Treatment of Stress Urinary Incontinence
}

\author{
Zhe Wang, ${ }^{1,2}$ Yan Wen,, Yan Hui Li, ${ }^{1,3}$ Yi Wei, ${ }^{1}$ Morgaine Green,, Prachi Wani, \\ Pengbo Zhang, Renee Reijo Pera, and Bertha Chen ${ }^{1}$
}

There is great interest in using stem cells (SC) to regenerate a deficient urethral sphincter in patients with urinary incontinence. The smooth muscle component of the sphincter is a significant contributor to sphincter function. However, current translational efforts for sphincter muscle restoration focus only on skeletal muscle regeneration because they rely on adult mesenchymal SC as cell source. These adult SC do not yield sufficient smooth muscle cells (SMCs) for transplantation. We may be able to overcome this limitation by using pluripotent stem cell (PSC) to derive SMCs. Hence, we sought to investigate whether smooth muscle precursor cells (pSMCs) derived from human PSCs can restore urethral function in an animal model generated by surgical urethrolysis and ovariectomy. Rats were divided into four groups: control (no intervention), sham saline (surgery + saline injection), bladder SMC (surgery + human bladder SMC injection), and treatment (surgery + pSMC injection, which includes human embryonic stem cell (hESC) H9-derived pSMC, episomal reprogrammed induced pluripotent stem cells (iPSCs)derived pSMC, or viral reprogrammed iPSC-derived pSMC). pSMCs $\left(2 \times 10^{6}\right.$ cells/rat $)$ were injected periurethrally 3 weeks postsurgery. Leak point pressure (LPP) and baseline external urethral sphincter electromyography were measured 5 weeks postinjection. Both iPSC-derived pSMC treatment groups showed significantly higher LPP compared to the sham saline group, consistent with restoration of urethral sphincter function. While the difference between the H9-derived pSMC treatment and sham saline group was not significant, it did show a trend toward restoration of the LPP to the level of intact controls. Our data indicate that pSMCs derived from human PSCs (hESC and iPSC) can restore sphincter function.

\section{Introduction}

$\mathrm{S}$ TRESS URINARY INCONTINENCE (SUI) is a major health $\checkmark$ problem with significant social and economic impact [1]. SUI is associated with a deficient urethral sphincter that cannot generate sufficient closure pressure to resist the increase in abdominal pressure during physical activity. Current treatment options include pelvic floor exercise [2], bulking agent injections into the urethral tissues, or surgery with placement of permanent mesh [3-5]. The injection of bulking agents is less invasive than surgery, but achieves variable short-term results. The need for repeat injections and persistent leakage limits its overall success. The surgical midurethral sling is efficacious for the majority of patients, but not everyone is responsive and recurrent leakage can occur years after the initial surgery, leaving a population of patients with few other options.

The urethral sphincter relies on mechanical integrity of striated and smooth muscle cells (SMCs) for effective urethral closure and continence [6]. The internal urethral sphincter, consisting of primarily SMCs, is a significant contributor to overall continence. Currently there is no therapy to restore function to this portion of the urethra. Stem cell (SC)-based therapy is attractive because it is thought that SCs can replace the specific cell types necessary to restore function. Various sources of SCs have been studied. Mesenchymal stem cells utilized in several studies were obtained from a variety of adult and fetal tissues [5-8]. So far, these adult mesenchymal stem cells have yielded promising results in preclinical and animal studies [9-15]. The

\footnotetext{
${ }^{1}$ Department of OB/GYN, Stanford University School of Medicine, Stanford, California.

${ }^{2}$ The Center for Reproductive Medicine, OB/GYN, Nanfang Hospital, Southern Medical University, Guangzhou, Guangdong, People's Republic of China.

${ }^{3}$ The Department of OB/GYN, Union Hospital, Tongji Medical College, Huazhong University of Science and Technology, Wuhan, People's Republic of China.

${ }_{5}^{4}$ Department of Pathology, Stanford University School of Medicine, Stanford, California.

${ }^{5}$ Department of Cell Biology and Neurosciences and Department of Chemistry and Biochemistry, Montana State University, Bozeman, Montana.
} 
SC-like cells for transplantation obtained from these adult tissues contain a heterogeneous mixture of mesenchymal cells, such as myoblasts at various stages of differentiation originating from satellite cells, mesenchymal stem cells, striated muscle cells, and fibroblasts. Some studies show that only a small fraction of the injected mesenchymal stem cell mixture ultimately differentiate into SMCs in situ $[12,16]$. Therefore, there are insufficient cells destined specifically to restore the smooth muscle component of the sphincter. Furthermore, all these cells possess senescence and epigenetic changes from the adult donor, which limits the total number of cells that can be harvested and expanded from the tissue biopsies.

Human embryonic stem cells (hESCs) are pluripotent stem cells (PSCs) capable of self-renewal and differentiation into all three embryonic (ectoderm, mesoderm, and endoderm) lineages. They are also devoid of senescence and have minimal epigenetic changes, suggesting that they may be an improved $\mathrm{SC}$ source for regenerative therapies. Evolving technologies have enabled the genetic reprogramming of somatic cells into induced pluripotent stem cells (iPSC), which possess many of the PSC properties. This has opened up a new cell source for autologous SC therapies. Therefore, in this study, we sought to investigate whether PSCs such as hESCs and patient-specific iPSCs can be used as a SC source for derivation of a pure population of smooth muscle precursor cells (pSMCs) and whether transplantation of these precursor cells can restore urethral function in a SUI rat model.

\section{Materials and Methods}

The Institutional Review Board of the Stanford University School of Medicine and the Stanford University Stem Cell Research Oversight Committee approved this study. The animal protocols were approved by the Stanford Animal Research Committee. A total of 108 adult female Virgin, Rowett Nude (RNU) rats, ages 11-12 weeks were used for this study.

\section{Bilateral ovariectomy (bilateral salpingo-oophorectomy)}

A $1.5-2 \mathrm{~cm}$ low-vertical abdominal incision is made in the rat. The ovaries are exteriorized through the abdominal incision by grasping the periovarian fat. A 4-0 absorbable suture is used to ligate the junction between the fallopian tube and the ovary together with all the accompanying blood vessels and fat. The ovaries are transected and the fallopian tubes are returned into the abdominal cavity.

\section{Urethrolysis}

Urethrolysis is performed as described by Rodriguez et al. [17], except with a minor modification. The fatty tissue in front of the bladder neck, rather than the bladder, is pulled during the urethral dissection. We introduced this modification to minimize trauma to the bladder. The proximal and distal urethra is detached circumferentially by incising the endopelvic fascia and separating the urethra from the anterior vaginal wall and pubic bone by sharp dissection.

\section{Cell preparation}

Human bladder smooth cells (passage 3; LONZA) are used as terminally differentiated cell treatment for comparison with pSMC treatment. They are cultured in $90 \%$
Dulbecco's modified Eagle's medium and $10 \%$ fetal bovine serum (Fisher Scientific) at $37^{\circ} \mathrm{C}$ in an atmosphere of $95 \%$ air and $5 \% \mathrm{CO}_{2}$. Cells are transplanted into the animals at passage 7 .

Human pSMCs are differentiated from hESCs [luciferase (Fluc)-tagged H9 line, a gift from Dr. Joseph Wu, Stanford University], retrovirus reprogrammed PSCs (iPSCs), and episomal vector reprogrammed PSCs (Epi-iPSCs). The iPSC (a gift from Dr. Michele Pamela Calos, Stanford University) and Epi-iPSC lines were reprogrammed from fibroblasts from two separate donors. The reprogramming methodology is as described by Takahashi et al. and Nguyen et al. [18,19] and by $\mathrm{Yu}$ et al. [20], respectively. G-banded karyotyping of iPSC was performed as previously described by WiCell [21]. To track cells by imaging techniques, a fraction of the pSMCs is differentiated from SCs transfected with modified attB donor plasmid encoding luciferase gene. Luciferase (Luc)-tagged progenitor smooth cells were used for in vivo tracking.

Human pSMCs are differentiated from iPSCs and ESCs (H9) using a modified, feeder cell-free, vascular progenitor protocol as previously described [22,23]. Briefly, the SCs are cultured in chemically defined media [RPMI 1640 with $1 \mathrm{mM}$ Glutamax, $1 \%$ Nonessential Amino Acids, $0.1 \mathrm{mM} \beta-$ mercaptoethanol, $1 \%$ penicillin and streptomycin (Life Science Technology, Inc.)], $1 \%$ ITS (Sigma-Aldrich) supplemented with Activin A (50 ng/mL), BMP4 (50 ng/mL, Sigma), then basic fibroblast growth factor $(50 \mathrm{ng} / \mathrm{mL})$, and vascular endothelial growth factor ( $40 \mathrm{ng} / \mathrm{mL}$; Invitrogen). The cells are then sorted for CD31+/CD34+ vascular progenitors by FACS. Sorted cells are expanded in SMC growth medium (Invitrogen) to yield pSMCs. The subset of these precursor cells are allowed to terminally differentiate to mature SMCs for further characterization and functional tests. pSMC are characterized by staining for $\alpha$-SMA, CNN1, SM22 $\alpha$, smoothelin, and desmin. Ki67 staining is used to show proliferation [24]. Terminal SMCs are characterized by staining for myosin heavy chain and elastin and also by contraction assay. Contractions are induced by treating the cells with $100 \mathrm{mM}$ Carbachol (Sigma-Aldrich). Images are recorded every minute for $10 \mathrm{~min}$ on a Nikon BioStation IM (Nikon; www.nikon.com) [22].

\section{Implantation of human pSMCs into rodents}

The animals are anesthetized with $1 \%-3 \%$ isoflurane. To examine human pSMC survival in vivo, 1 million luciferasetagged pSMCs in $50 \mu \mathrm{L}$ of Matrigel $(50 \%)$ are injected directly into the adductor longus muscles of five SCID mice and imaged for 3 months. To examine the effect of pSMCs on the restoration of urethral function in SUI rats, cells suspended in $100 \mu \mathrm{L}$ of smooth muscle growth supplement (Life Technologies) are injected periurethrally three weeks after urethrolysis. We use a commercial, terminally differentiated human SMC line (passage 7; LONZA) for comparison with the pSMC treatment. A total of $2 \times 10^{6}$ cells suspended in the respective medium are injected into each urethra. The sham treatment SUI rat (urethrolysis plus ovariectomy) is injected with saline only.

Two experiments were performed, one testing pSMCs derived from hESCs H9 line (H9-pSMC) and another testing pSMCs derived from human iPSC (iPSC-pSMC) and EpiiPSC lines (Epi-iPSC-pSMC). Rodent experimental groups are shown in Table 1. 
Table 1. Rodent Groups in Each Experiment

\begin{tabular}{lcccc}
\hline Experiment & Control $(N)$ & Sham saline $(N)$ & H9-pSMC $(N)$ & Bladder-SMC $(N)$ \\
\hline H9-pSMC experiment & 13 & 12 & 22 & 11 \\
iPSC-pSMC experiment & Control $(N)$ & Sham saline $(N)$ & Epi-iPSC-pSMC $(N)$ & iPSC-pSMC $(N)$ \\
\hline
\end{tabular}

Epi-iPSC, episomal vector reprogrammed PSC; pSMC, smooth muscle precursor cell; iPSC, induced pluripotent stem cell.

\section{In vivo bioluminescence imaging of transplanted pSMCs}

Luc-tagged pSMCs $\left(2 \times 10^{6}\right)$ are injected into the urethral sphincter region of the RNU rats or the hind leg of SCID mice for in vivo cell tracking. Transplanted cell survival is longitudinally monitored through bioluminescence imaging (BLI) using the Xenogen In Vivo Imaging System (Caliper Life Sciences). In brief, D-luciferin (BIOSYNTH INTERNATIONAL, Inc.) is administered intraperitoneally at a dose of $375 \mathrm{mg} / \mathrm{kg}$ of body weight $15 \mathrm{~min}$ before image acquisition. Animals are placed in a light-tight chamber, and photons emitted from luciferase-expressing cells are collected with integration times of $2 \mathrm{~min}$. BLI signal is quantified in maximum photons $/ \mathrm{s} / \mathrm{cm}$ per steradian and presented as $\log _{10}$ (photons/s). To examine the viability of transplanted cells in the sphincter urethra of rats or the hind leg of mice, the images are obtained every 2 days until disappearance of signals, then once per week for 5 weeks or 3 months, respectively.

\section{Double immunofluorescence staining}

The cryostat sections of mouse hind leg muscle are prepared and fixed as described previously [25]. The slides are treated with $0.25 \%$ Triton X-100 in phosphate-buffered saline (PBS) for $10 \mathrm{~min}$ at room temperature. After washing with phosphate-buffered saline with $0.01 \%$ Tween 20 (PBS-T)$(0.01 \%$ Tween in PBS) and blocking with $1 \%$ bovine serum albumin in PBS-T, the slides are double stained with $1 / 50$ rabbit anti- smoothelin (Santa Cruz Biotechnology) and 1/50 mouse anti-human nuclei (HuNu; EMD Millipore Corporation) primary antibody at $4^{\circ} \mathrm{C}$ overnight. Then after washing, the slides are incubated with $1 / 100$ mouse anti-rabbitimmunoglobulin $\mathrm{G}$ (IgG)-fluorescein isothiocyanate and 1/100 goat anti-mouse-IgG-tetramethylrhodamineisothiocyanate (Sigma) at room temperature for $1 \mathrm{~h}$ in a dark chamber. The slides of non-cell injected hind leg muscle from the same mouse are used as negative controls. The 4',6-diamidine2-phenylidole is used to stain the nuclei. After rinsing and mounting, the slides are examined with a fluorescence microscope.

\section{Suprapubic bladder catheter implantation}

Five weeks after injection, rats are anesthetized for leak point pressure (LPP) measurements. A suprapubic incision is made and the bladder exteriorized from the abdominal cavity. A small incision is made in the dome of the bladder and a polyethylene catheter (PE-50 tube with a flared tip) (Becton Dickinson) is inserted into the bladder. The catheter is connected through a three-way stopcock to a syringe for filling with physiological saline and to a pressure transducer (TSD 104A; BIOPAC Systems, Inc.) for monitoring bladder pressure.

\section{Baseline measurement of urethral sphincter electromyography}

The 0.003" platinum-iridium wire electrodes (A-M Systems) are inserted into the mid urethra bilaterally near the urethral meatus using a $25 \mathrm{G} 5 / 8^{\prime \prime}$ syringe needle (BD Bioscience; Fig. 1). Electromyography (EMG) activity is recorded with a MP150 system (BIOPAC Systems). All EMG signals are recorded at $1,000 \mathrm{~Hz}$ and analyzed with the AcqKnowledge 3.9.1 software (BIOPAC Systems). We calculate the average rectified EMG [average rectified value (ARV)], which is defined as a time-windowed mean of the absolute value of the signal. ARV is one of the processing methods used to construct derived signals from raw EMG data and it is a measurement that can be used to analyze EMG data [26,27]. Three randomly selected ten-second segments at baseline bladder pressure are averaged to arrive at the mean of ARV.

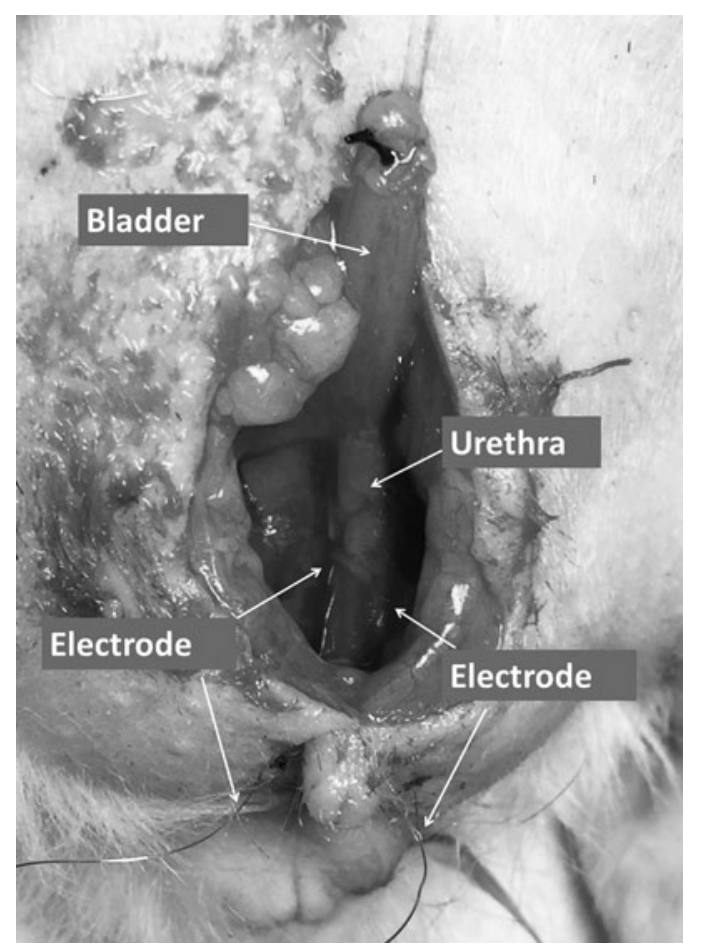

FIG. 1. Position of the electrodes for urethral sphincter EMG measurements. Electrodes are placed at the midurethra bilaterally, but inserted externally. EMG, electromyography. 


\section{Vertical tilt table LPP measurement}

Vertical tilt table LPP test is performed according to a previously described method [28]. Investigators performing LPP are blinded to the group assignment of each animal. Spinal cord transection is performed at the T8-T9 level to eliminate spontaneous bladder activity in response to increasing intravesical pressure. The rats are mounted on a tilt table and placed in a vertical position. The bladder catheter is connected to a $50-\mathrm{mL}$ syringe (reservoir), which is fixed onto a metered vertical pole. Intravesical pressure is elevated by raising the height of the reservoir gradually, by $2-3 \mathrm{~cm}$ increments every $2 \mathrm{~min}$ starting from $0 \mathrm{~cm}$, until urinary leakage is observed. The pressure at which leakage occurs is recorded as the LPP. The average of three consecutive LPPs from each rat is used for data analysis.

\section{Data statistical analysis}

Quantitative values are presented as the mean \pm standard error. JMP pro 10.0.2 software (SAS Institute, Inc.) is used for statistical analysis. Nonparametric comparisons for each pair are performed using the Wilcoxon Method, with $P<0.05$ indicating a significant difference between groups.

\section{Results}

\section{Characterization of terminally differentiated SMC}

Our differentiation protocol successfully differentiated pSMCs from human PSC lines (both hESCs and iPSCs). Immunofluorescence staining revealed robust expression of SM22 $\alpha, \alpha$-SMA, CNN1, smoothelin, and desmin in pSMCs. Terminal SMCs were characterized and exhibited expression of myosin heavy chain and elastin. Over $50 \%$ of cells contracted with carbachol stimulation consistent with human aorta SMCs (data not shown). Karyotype analysis revealed normal chromosomes in both iPSC lines, confirming that the genetic reprograming did not cause significant genetic aberrations.

\section{In vivo tracking of fluc-tagged $p S M C$}

Rats with periurethral injection of 2 million Fluc-tagged pSMCs showed a strong BLI signal in the urethral region $20 \mathrm{~min}$ after injection confirming site-appropriate injection (Fig. 2). BLI signal in the periurethral region of the SUI rats disappeared after 9 days. This may be due to cell attrition to a level below the detection level of BLI technique. This is because the RNU rat is not completely immunodeficient, thus causing the transplanted human cells to undergo attrition over time. Therefore, we used SCID mice to further examine cell survival and integration in vivo. Additionally, the deep location of the rat urethra decreases the intensity of the BLI signals. The SCID mouse is severely immunodeficient and injection into the hind leg, which is superficial, allows for better detection of the BLI signal. BLI signal of iPSC-pSMCs injected into the hind leg of the SCID mice persisted for 2043 days. Re-emergence of the BLI signal was observed and the signal persisted for at least 88 days when the experiment was terminated (Fig. 3). Cells in the skeletal muscle of the SCID mouse stained positive for $\mathrm{HuNu}$ (protein) and human smoothelin (a SMC protein) confirmed persistence of human SMCs in vivo (Fig. 3).

\section{H9-pSMC efficacy experiment}

Average LPP value of the sham saline injection group was significantly lower $\left(N=10\right.$, mean $\left.=15.52 \pm 1.09 \mathrm{~cm} \mathrm{H}_{2} \mathrm{O}\right)$ than intact controls $\left(N=13\right.$, mean $\left.=18.75 \pm 0.96 \mathrm{~cm} \mathrm{H}_{2} \mathrm{O}, P<0.05\right)$ 8 weeks after urethrolysis, confirming persistence of SUI in the model. The average LPP value of the bladder SMC group $\left(N=11\right.$, mean $\left.=18.95 \pm 1.04 \mathrm{~cm} \mathrm{H}_{2} \mathrm{O}\right)$ was significantly higher than that of the sham saline group $(P<0.05)$. LPP value of the H9-pSMC treatment group was higher than that of the saline sham group, although the difference was not significant. There was also no difference in LPP between the H9-pSMC treatment group $\left(N=22\right.$, mean $\left.=16.66 \pm 0.74 \mathrm{~cm} \mathrm{H}_{2} \mathrm{O}\right)$ and intact controls (Fig. 4). The group treated with human SMCs showed significant improvement in LPP compared to the sham saline group. Two outlier LPP values $\left(22 \mathrm{~cm}\right.$ of $\mathrm{H}_{2} \mathrm{O}$ and $2.3 \mathrm{~cm}$ of $\mathrm{H}_{2} \mathrm{O}$ ) were deleted in the sham saline group. It is not uncommon for RNU rats to have variable anatomy due to inbreeding [29]. Therefore, extremes in measurements are likely to reflect anatomic variance and may skew the data abnormally.

\section{iPSC-pSMC efficacy experiment}

SUI models again demonstrated persistence of SUI throughout the experiment according to the LPP comparison between the intact control group $\left(N=13\right.$, mean $\left.=17.67 \pm 1.11 \mathrm{~cm} \mathrm{H}_{2} \mathrm{O}\right)$ and sham saline group $\left(N=14\right.$, mean $=14.16 \pm 1.07 \mathrm{~cm} \mathrm{H}_{2} \mathrm{O}$, $P<0.05)$. Epi-iPSC-pSMC treatment group had significantly
FIG. 2. $2 \times 10^{6}$ Fluc-taggedH9-pSMC were transplanted into the urethra of the Rowett Nude rat and monitored by BLI. The BLI signal was strong on the day of transplantation and gradually became weaker until day 9 , when no signal was detected. BLI, bioluminescence imaging; pSMC, smooth muscle precursor cell.

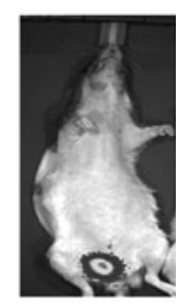

Day 0

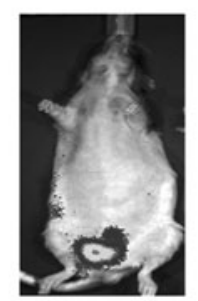

Day 2
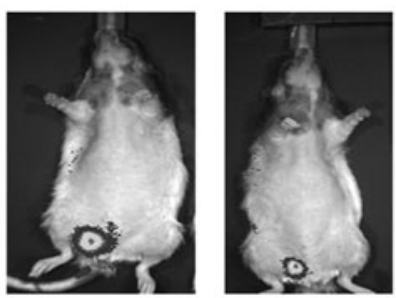

Day 5
Day 7

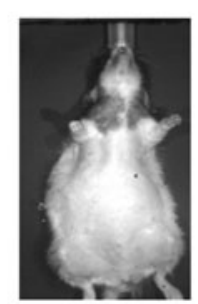

Day 9

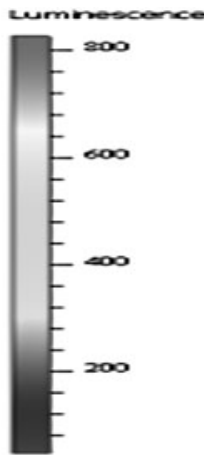

comes 

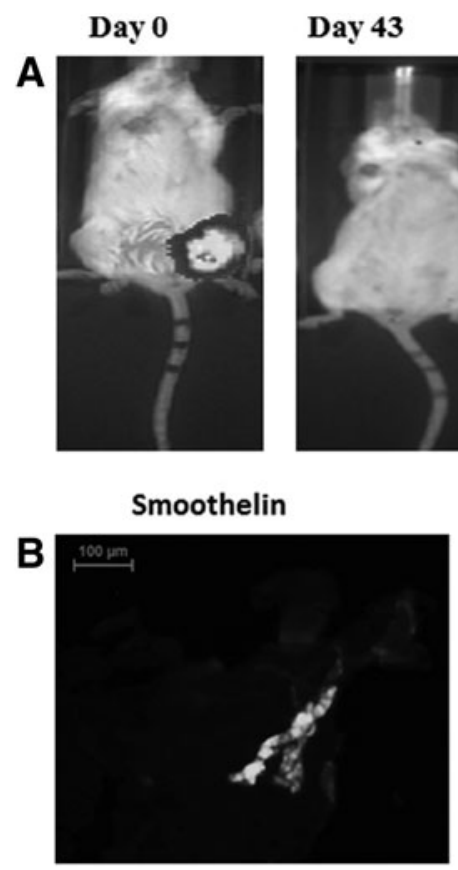

Smoothelin/DAPI

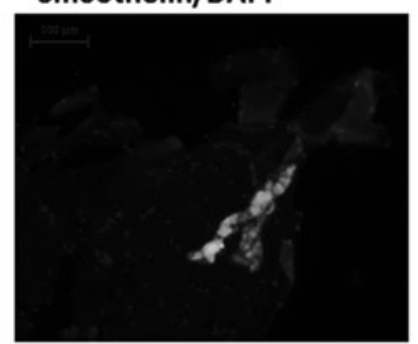

Day 88

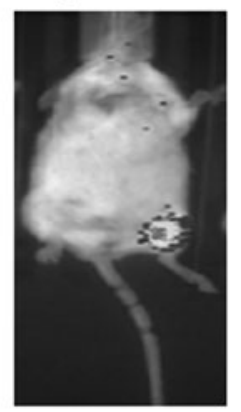

HuNuclei

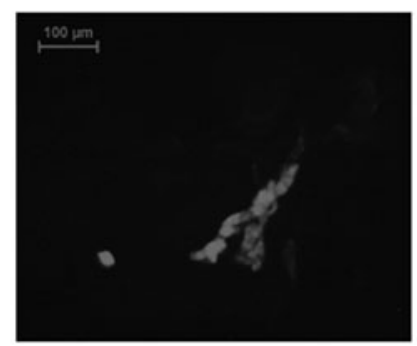

HuNuclei/DAPI

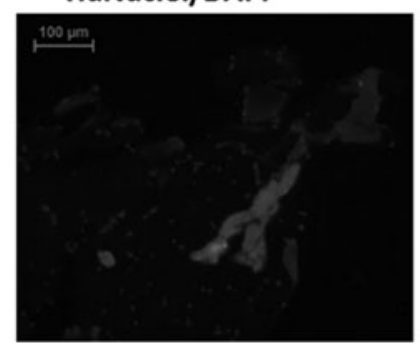

Smoothelin/HuNuceli

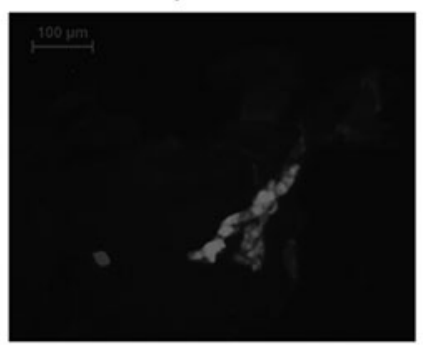

DAPI

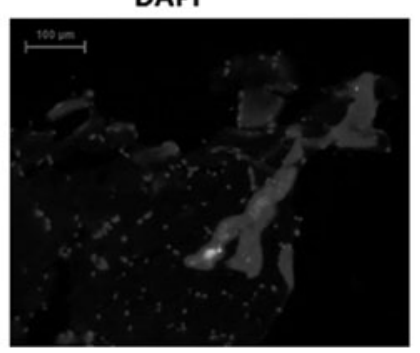

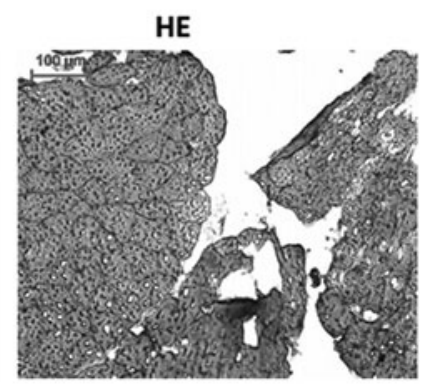

FIG. 3. (A) The SCID mouse injected with luciferase-tagged iPSC-pSMCs $\left(1 \times 10^{6}\right)$ in the one hind leg was monitored with BLI for 88 days. The BLI signal disappeared at day 43, and then reappeared at day 88. (B) Identification of the injected iPSC-pSMCs in the hind leg of SCID mouse with immunofluorescence staining. At day 88 after cell injection, the transplanted cells, which were stained positive with human nuclei (HuNuclei) and human smoothelin, were detected focally at the injection site of the hind leg. (Scale bar: $100 \mu \mathrm{M})$. iPSC, induced pluripotent stem cell.

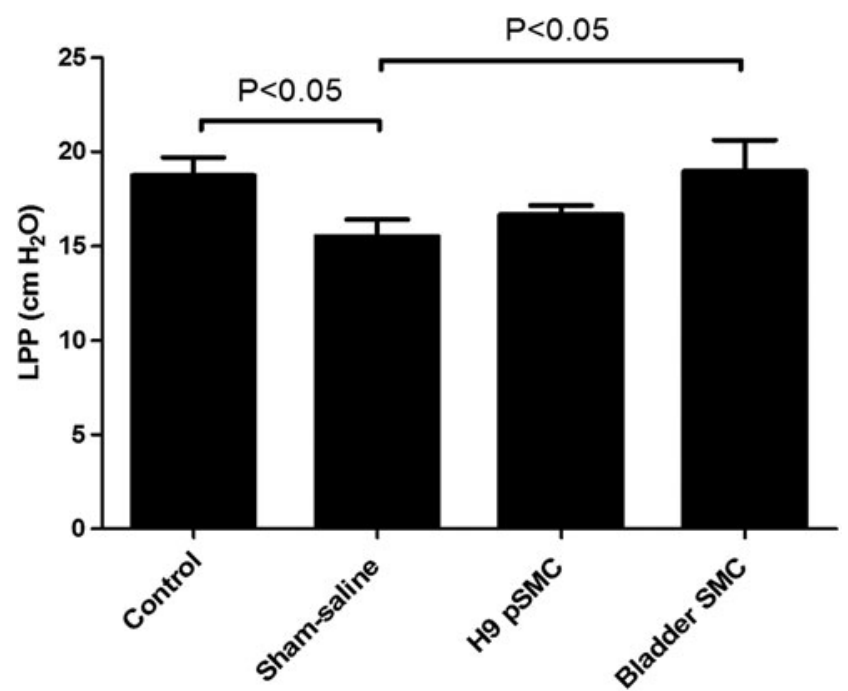

FIG. 4. Comparison of LPP values between groups in the H9-pSMC efficacy experiment. The results are presented as the mean \pm standard error of mean. LPP, leak point pressure. higher LPP value $\left(N=9\right.$, mean $\left.=19.4 \pm 1.33 \mathrm{~cm} \mathrm{H}_{2} \mathrm{O}\right)$ compared to sham saline group $(P<0.05)$. LPP value of iPSC-pSMC group was also significantly higher $(N=8$, mean $=18.45 \pm 1.41 \mathrm{~cm}$ $\left.\mathrm{H}_{2} \mathrm{O}\right)$ than the sham saline group $(P<0.05$, Fig. 5). Extreme outlier LPP values were also observed in each group, including in intact controls. These outliers were removed from our data analysis because of concerns related to anatomic variability.

\section{Baseline EMG activities of the urethral sphincter}

EMG activity represents the motor unit potential of the sphincter muscle. SMCs have resting EMG, therefore, the recorded EMG activity includes that of both skeletal and smooth muscles [30]. EMG measurements can provide information about the integrity of innervation and the condition of neuromuscular junctions and myofibers [31]. The average rectified EMG (ARV) reflects the compound action potential amplitude level. The greater the amplitude of the ARV, the greater the number of excited muscle cells. ARVs were used to compare EMG activity between the experimental groups (Fig. 6). 


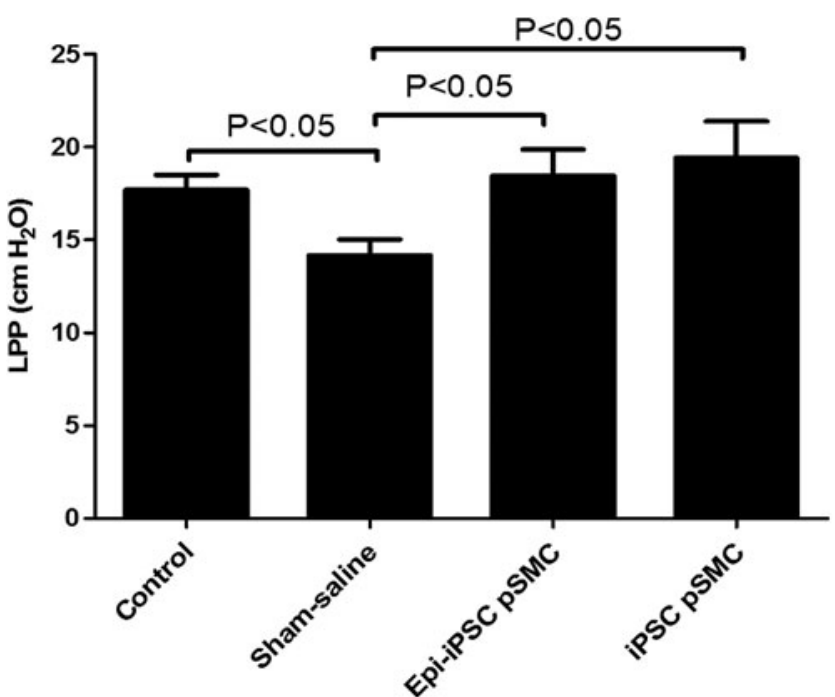

FIG. 5. Comparison of LPP values between groups in the iPSCs-pSMC efficacy experiment. The results are presented as the mean \pm standard error of mean.

$\mathrm{ARV}$ in the control group $(N=11$, mean $=0.000383 \pm$ $0.00008 \mathrm{mV})$ was significantly higher than in the sham saline group $(N=12$, mean $=0.00017 \pm 0.000076 \mathrm{mV}, P<0.05)$ and bladder SMC group $(N=11$, mean $=0.000185 \pm 0.00008 \mathrm{mV}$, $P<0.05)$. This is consistent with the surgical neuromuscular injury the sham saline and bladder SMC groups experienced.
Although the ARV for the H9-pSMC treatment group $(N=22$, mean $=0.000299 \pm 0.000056 \mathrm{mV})$ appeared higher than the sham saline group, the difference was not statistically significant (Fig. 7). EMG data from all but two rats in the control group were available for analysis. Data loss was due to software issues.

Both Epi-iPSC-pSMC treatment group $(N=10$, mean $=$ $0.000158 \pm 0.000031 \mathrm{mV})$ and iPSC-pSMC treatment group $(N=6$, mean $=0.000119 \pm 0.00004 \mathrm{mV})$ showed higher ARV trends compared to the sham saline group (Fig. 8). ARV data of four rats in the sham saline group and three rats in the iPSC-pSMC group were lost because data were not archived correctly in the system.

Overall, we found that placement of the wire electrodes in the rat periurethral region was difficult and unreliable.

\section{Discussion}

Urethral sphincter muscle, connective tissue, neural, and vascular support are responsible for maintaining continence [32]. The smooth muscle component has been documented to be an important contributor to urethral pressure recovery and leakage prevention in animal studies [33]. It is thought that the internal urethral sphincter, which consists of SMCs, is an important contributor to urinary continence. Weakening of this area due to injury or loss of cells from aging is associated with moderate to severe incontinence [34-36]. Current therapies do not address restoration of SMCs in the urethral sphincter.

A

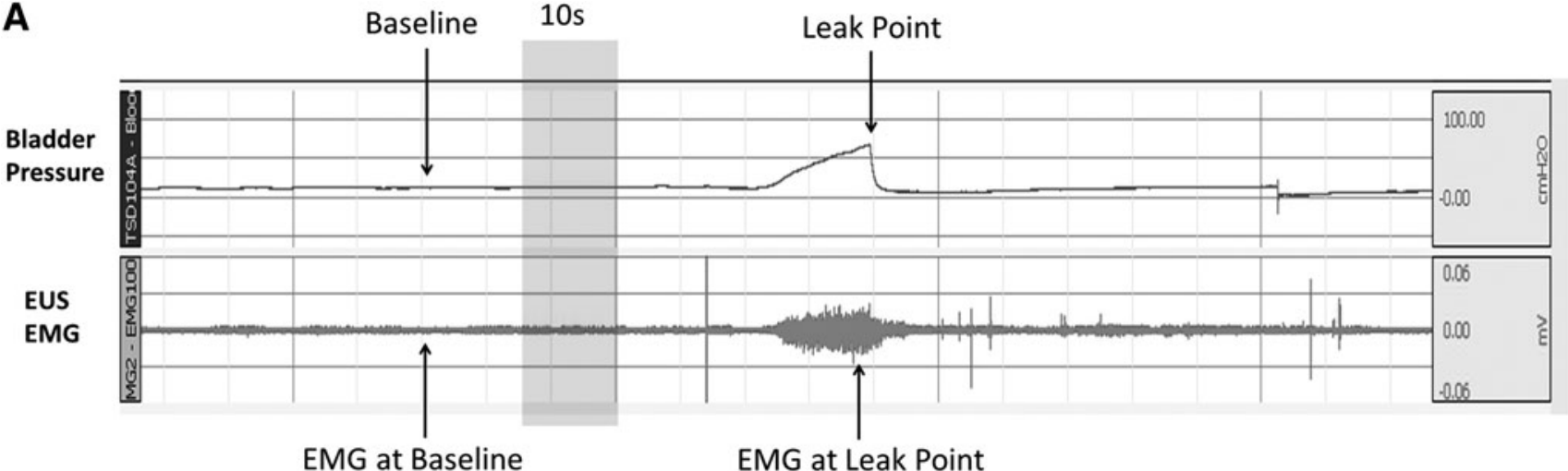

B

EMG at Baseline

EMG at Leak Point

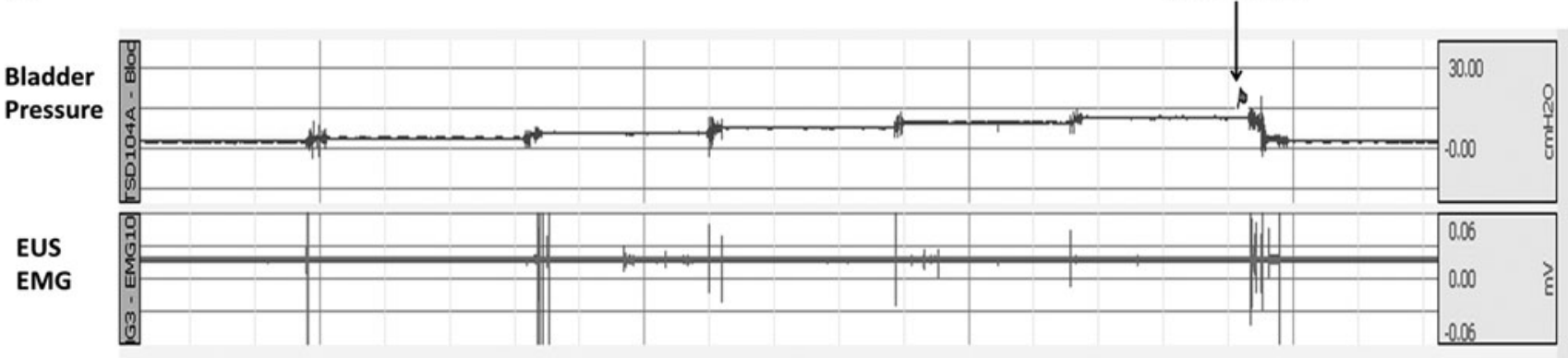

FIG. 6. (A) A representative EMG activity tracing from a control rat. Baseline EMG was stable when bladder pressure was near $0 \mathrm{~cm} \mathrm{H}_{2} \mathrm{O}$. The arrow (EMG at baseline) indicates a randomly selected $10 \mathrm{~s}$ segment used for ARV analysis. The EMG at leak point arrow shows the variability of the measurement at LPP. (B) A representative LPP graph from a control rat after spinal transection. Intravesical pressure was elevated by raising the height of reservoir gradually by $2-3 \mathrm{~cm}$ every 2 min. The arrow indicates when urinary leakage was observed. No bladder contraction was detected. ARV, average rectified value. 


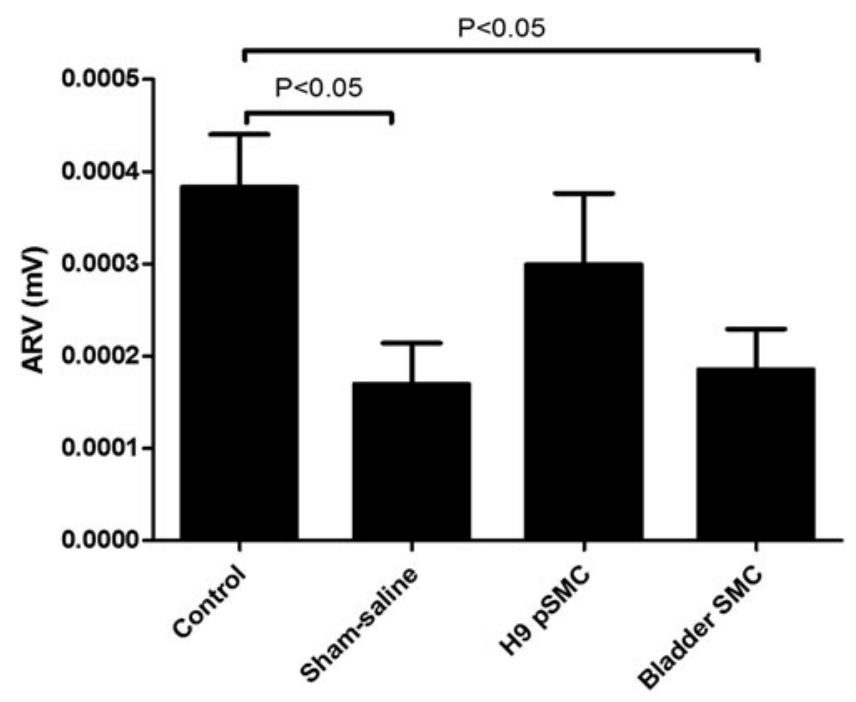

FIG. 7. Comparison of EMG (ARV) between groups in the H9-pSMC efficacy experiment. The results are presented as the mean \pm standard error of mean.

In recent years, SC-based regenerative medicine has shown promising effects on the recovery of human sphincter dysfunction or injury. In these studies, implanted SCs were urine derived [13] or adult muscle biopsy derived [37], which consisted of heterogeneous cells with small numbers of SC subpopulations. Hence, the effect for SUI recovery was generated by a mixture of cells with variable differentiation and renewal potential. It is impossible to correlate the efficacy for functional regeneration of the urethral sphincter to specific cell types. In this study, we used a pure population of pSMCs differentiated from human PSCs, hESC, and iPSC, to investigate its effect on SUI recovery.

In both of our experiments (using H9- and iPSC-pSMCs), LPP was significantly lower in the sham saline (surgery + saline injection) group compared to intact controls, confirming persistence of SUI in our animal model throughout the experi-

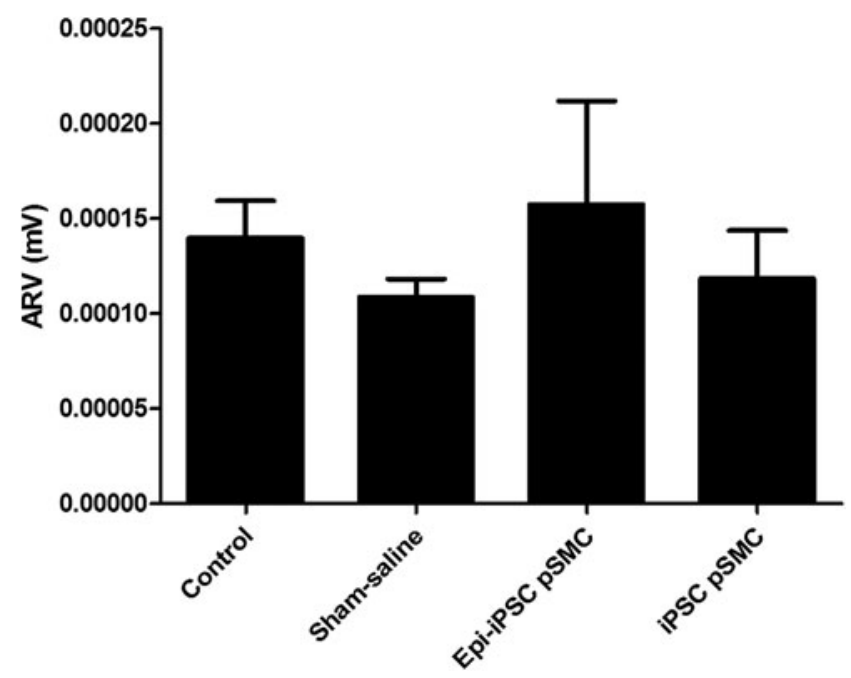

FIG. 8. Comparison of EMG (ARV) between groups in the iPSC-pSMC efficacy experiment. The results are presented as the mean \pm standard error of mean. No significant differences were found. mental period. While there was no significant difference between the H9-pSMC treatment and the sham saline group, LPP was higher in the former and trended toward that of intact controls, suggesting restoration of urethral function. Data from the iPSC-pSMC experiment did show significant improvement in LPP for both iPSC-pSMC and Epi-iPSC-pSMC treatment groups compared to the sham saline group. Because LLP measurements are prone to observer bias, the researchers in this study were blinded to the rat treatment groups.

We note that a change in cell processing protocol may have contributed to the lack of significance in the H9-pSMC study. The H9-pSMCs were freshly thawed before transplantation. Whereas, cells for the iPSC-pSMC study were allowed to recover in culture for 2 days after thawing and before transplantation. This could have contributed to improved in vivo performance in the iPSC experiment. Another potential source of variability relates to the RNU rats. They are known to have variable anatomy due to inbreeding [29]. This can account for the extreme outlier values seen even in the control group. To minimize this effect, we deleted the extreme outliers in our analysis.

Bladder SMCs as a source of SMCs for bladder tissue regeneration has been studied [38-41]. The bladder SMCs and urothelial cells were reported to contribute to the development of bladder smooth muscle [42]. However, biopsy barriers and severe fibrosis were significant issues. We used bladder SMCs to explore whether transplantation of terminally differentiated SMCs can restore urethral function in our SUI rat model. In our study, LPP was significantly higher with bladder SMCs treatment compared to the sham saline injected rats, confirming that replacement of SMCs restores function. However, autologous bladder SMC transplantation and autologous muscle-biopsy SC therapies share similar limitations, including biopsy barriers, ability to harvest sufficient number of cells, limited cell expansion capabilities, and cell senescence.

EMG activity has been used to assess sphincter function $[30,43]$ and evaluate its regeneration in some studies involving autologous muscle progenitor cell and periurethral skeletal myofiber treatment $[44,45]$.

Therefore, we included EMG measurements as an adjunct to our LPP data. Our primary outcome measure is LPP. In our study, pSMC-treatment groups showed increases in ARV, but the differences were not significant compared to the sham saline group. Our LPP data does not agree with the EMG data. We believe this is due to the high variability of this methodology and the loss of data related to technical issues. The rat urethral sphincter measures roughly $1 \mathrm{~cm}$ in length, which makes it difficult to insert electrodes externally into the same area reproducibly (Fig. 1). Thus, inadequate EMG activity or excessive crosstalk from other muscles can cause high variability [46]. We did not use the EMG activity at leak point because that measurement is subject to even more uncertainty (Fig. 6A). The high variability makes EMG unsuitable as a measurement method for studies involving many animals. Our experience agrees with other publications, where LPP is used as the primary measurement method to assess urethral function.

There are several possible ways in which pSMCs may restore urethral function: (i) differentiation of pSMCs into functional SMCs, (ii) pSMCs may persist in the urethra acting as a bulking agent, and/or (iii) autocrine and/or 
paracrine effects of secreted cytokines that induce proliferation of rat urethral sphincter muscles [47] or extracellular matrix. According to the BLI tracking of pSMCs in vivo, signals disappeared in all rats after 9 days, suggesting that there was significant attrition of the transplanted pSMCs 1 week postinjection. Perhaps the pSMCs could not survive the stress of transplantation or they were eliminated by the incompletely suppressed immune system of the RNU rats. However, given the LPP results, it is possible that a small population of pSMC, too low to be detected by BLI, was able to expand and continue to differentiate into terminal SMCs. To test this hypothesis, we conducted a cell integration study using SCID mice. This type of mouse is severely immunodeficient. We also injected into a muscle area that is more superficial to increase ability to detect the BLI signal. Our data confirm survival of pSMCs in vivo up to 88 days and further in vivo differentiation into SMCs. Hence, cell integration is part of the pSMC mechanism of action post transplantation. We are currently conducting studies to examine the paracrine effects of pSMCs.

\section{Conclusion}

This is a translational study that explores a unique SC source for SMC regeneration. Currently most therapies for muscle replacement focus on skeletal muscle regeneration through adult mesenchymal stem cells. There are no treatments that target replacement of SMCs, which are a significant component of many organs. The positive trend in LLP with hESC, H9pSMC transplant, and significant improvements in LLP with iPSC-pSMC transplant, in conjunction with evidence of longterm cell integration, suggest that human PSC-derived pSMCs can restore urethral sphincter function. These findings open up the possibility of using PSCs for urologic conditions, where large numbers of SMCs are needed to restore function.

\section{Acknowledgments}

The authors would like to express their gratitude to Fangfang $\mathrm{Zhu}, \mathrm{PhD}$ (postdoctoral research fellow in Dr. Michele Pamela Calos' Laboratory, Stanford University School of Medicine) for her SC technical support. We would like to thank China Chien for her invaluable advice and help with the leak point pressure testing methodology. This research is funded by CIRM (California Institute of Regenerative Medicine), Grant No. 106180-TR3-05569.

\section{Author Disclosure Statement}

No competing financial interests exist.

\section{References}

1. Imamura M, P Abrams, C Bain, B Buckley, L Cardozo, J Cody, J Cook, S Eustice, C Glazener, et al. (2010). Systematic review and economic modelling of the effectiveness and cost-effectiveness of non-surgical treatments for women with stress urinary incontinence. Health Technol Assess 14:1-188, iii-iv.

2. Holroyd-Leduc JM, V Steinke, D Elliott, K Mullin, K Elder, S Callender and KA Hildebrand. (2013). Improving the quality of care for older adults using evidence-informed clinical care pathways. Can Geriatr J 16:111-113.
3. Hart ML, A Izeta, B Herrera-Imbroda, B Amend and JE Brinchmann. (2015). Cell therapy for stress urinary incontinence. Tissue Eng Part B Rev 21:365-376.

4. Sathianathen NJ, SM McGuigan and DA Moon. (2014). Outcomes of artificial urinary sphincter implantation in the irradiated patient. BJU Int 113:636-641.

5. Wang HJ, YC Chuang and MB Chancellor. (2011). Development of cellular therapy for the treatment of stress urinary incontinence. Int Urogynecol J 22:1075-1083.

6. Wallner C, NF Dabhoiwala, MC DeRuiter and WH Lamers. (2009). The anatomical components of urinary continence. Eur Urol 55:932-943.

7. Covas DT, RA Panepucci, AM Fontes, WA Silva, Jr., MD Orellana, MC Freitas, L Neder, AR Santos, LC Peres, MC Jamur and MA Zago. (2008). Multipotent mesenchymal stromal cells obtained from diverse human tissues share functional properties and gene-expression profile with CD146+ perivascular cells and fibroblasts. Exp Hematol 36:642-654.

8. Rodriguez LV, Z Alfonso, R Zhang, J Leung, B Wu and LJ Ignarro. (2006). Clonogenic multipotent stem cells in human adipose tissue differentiate into functional smooth muscle cells. Proc Natl Acad Sci U S A 103:12167-12172.

9. Cannon TW, JY Lee, G Somogyi, R Pruchnic, CP Smith, J Huard and MB Chancellor. (2003). Improved sphincter contractility after allogenic muscle-derived progenitor cell injection into the denervated rat urethra. Urology 62:958-963.

10. Carr LK, D Steele, S Steele, D Wagner, R Pruchnic, R Jankowski, J Erickson, J Huard and MB Chancellor. (2008). 1-Year follow-up of autologous muscle-derived stem cell injection pilot study to treat stress urinary incontinence. Int Urogynecol J Pelvic Floor Dysfunct 19:881-883.

11. Fu Q, XF Song, GL Liao, CL Deng and L Cui. (2010). Myoblasts differentiated from adipose-derived stem cells to treat stress urinary incontinence. Urology 75:718-723.

12. Lin G, G Wang, L Banie, H Ning, AW Shindel, TM Fandel, TF Lue and CS Lin. (2010). Treatment of stress urinary incontinence with adipose tissue-derived stem cells. Cytotherapy 12:88-95.

13. Liu G, X Wang, X Sun, C Deng, A Atala and Y Zhang. (2013). The effect of urine-derived stem cells expressing VEGF loaded in collagen hydrogels on myogenesis and innervation following after subcutaneous implantation in nude mice. Biomaterials 34:8617-8629.

14. Stangel-Wojcikiewicz K, D Jarocha, M Piwowar, R Jach, T Uhl, A Basta and M Majka. (2014). Autologous musclederived cells for the treatment of female stress urinary incontinence: a 2-year follow-up of a Polish investigation. Neurourol Urodyn 33:324-330.

15. Strasser H, R Marksteiner, E Margreiter, M Mitterberger, GM Pinggera, F Frauscher, M Fussenegger, K Kofler and G Bartsch. (2007). Transurethral ultrasonography-guided injection of adult autologous stem cells versus transurethral endoscopic injection of collagen in treatment of urinary incontinence. World J Urol 25:385-392.

16. De Coppi P, A Callegari, A Chiavegato, L Gasparotto, M Piccoli, J Taiani, M Pozzobon, L Boldrin, M Okabe, et al. (2007). Amniotic fluid and bone marrow derived mesenchymal stem cells can be converted to smooth muscle cells in the cryo-injured rat bladder and prevent compensatory hypertrophy of surviving smooth muscle cells. J Urol 177: 369-376.

17. Rodriguez LV, S Chen, GS Jack, F de Almeida, KW Lee and R Zhang. (2005). New objective measures to quantify 
stress urinary incontinence in a novel durable animal model of intrinsic sphincter deficiency. Am J Physiol Regul Integr Comp Physiol 288:R1332-R1338.

18. Takahashi K, K Tanabe, M Ohnuki, M Narita, T Ichisaka, K Tomoda and S Yamanaka. (2007). Induction of pluripotent stem cells from adult human fibroblasts by defined factors. Cell 131:861-872.

19. Nguyen HN, B Byers, B Cord, A Shcheglovitov, J Byrne, $P$ Gujar, K Kee, B Schule, RE Dolmetsch, et al. (2011). LRRK2 mutant iPSC-derived DA neurons demonstrate increased susceptibility to oxidative stress. Cell Stem Cell 8:267-280.

20. Yu J, K Hu, K Smuga-Otto, S Tian, R Stewart, II Slukvin and JA Thomson. (2009). Human induced pluripotent stem cells free of vector and transgene sequences. Science 324:797-801.

21. Seabright M. (1971). A rapid banding technique for human chromosomes. Lancet 2:971-972.

22. Marchand M, EK Anderson, SM Phadnis, MT Longaker, JP Cooke, B Chen and RA Reijo Pera. (2014). Concurrent generation of functional smooth muscle and endothelial cells via a vascular progenitor. Stem Cells Transl Med 3:91-97.

23. Wang C, X Tang, X Sun, Z Miao, Y Lv, Y Yang, H Zhang, P Zhang, Y Liu, et al. (2012). TGFbeta inhibition enhances the generation of hematopoietic progenitors from human ES cell-derived hemogenic endothelial cells using a stepwise strategy. Cell Res 22:194-207.

24. Loukovaara S, E Gucciardo, P Repo, J Lohi, P Salven and K Lehti. (2015). A case of abnormal lymphatic-like differentiation and endothelial progenitor cell activation in neovascularization associated with hemi-retinal vein occlusion. Case Rep Ophthalmol 6:228-238.

25. Chen X, DB Cho and PC Yang. (2010). Double staining immunohistochemistry. N Am J Med Sci 2:241-245.

26. Merletti R, M Knaflitz and CJ De Luca. (1990). Myoelectric manifestations of fatigue in voluntary and electrically elicited contractions. J Appl Physiol (1985) 69:1810-1820.

27. Lowery MM and MJ O'Malley. (2003). Analysis and simulation of changes in EMG amplitude during high-level fatiguing contractions. IEEE Trans Biomed Eng 50:1052-1062.

28. Conway DA, I Kamo, N Yoshimura, MB Chancellor and TW Cannon. (2005). Comparison of leak point pressure methods in an animal model of stress urinary incontinence. Int Urogynecol J Pelvic Floor Dysfunct 16:359-363.

29. Loh HS, MA Mohd-Lila, SO Abdul-Rahman and LJ Kiew. (2006). Pathogenesis and vertical transmission of a transplacental rat cytomegalovirus. Virol J 3:42.

30. Shafik AA, IA Shafik and O El Sibai. (2014). On the etiology of the electric activity of the external anal and urethral sphincters. J Invest Surg 27:267-272.

31. Rahman SW, KM Rahman, CB Sarker, SA Latif, S Ahmed, S Hossain and S Rahman. (2004). Neurophysiology in clinical practice. Mymensingh Med J 13:215-219.

32. Chen HY, YN Lin, YH Chen and WC Chen. (2012). Stress urinary incontinence following vaginal trauma involves remodeling of urethral connective tissue in female mice. Eur J Obstet Gynecol Reprod Biol 163:224-229.

33. Jiang HH, LB Salcedo and MS Damaser. (2011). Quantification of neurological and other contributors to continence in female rats. Brain Res 1382:198-205.

34. Clobes A, JO DeLancey and DM Morgan. (2008). Urethral circular smooth muscle in young and old women. Am J Obstet Gynecol 198:587 e1-e5.

35. Rodriguez L, M Carrillo, LA Sorbera, Y Zohar and S Zanuy. (2004). Effects of photoperiod on pituitary levels of three forms of GnRH and reproductive hormones in the male European sea bass (Dicentrarchus labrax, L.) during testicular differentiation and first testicular recrudescence. Gen Comp Endocrinol 136:37-48.

36. Trowbridge ER, JT Wei, DE Fenner, JA Ashton-Miller and JO Delancey. (2007). Effects of aging on lower urinary tract and pelvic floor function in nulliparous women. Obstet Gynecol 109:715-720.

37. Smaldone MC and MB Chancellor. (2008). Muscle derived stem cell therapy for stress urinary incontinence. World J Urol 26:327-332.

38. Deng K, DL Lin, B Hanzlicek, B Balog, MS Penn, MJ Kiedrowski, Z Hu, Z Ye, H Zhu and MS Damaser. (2015). Mesenchymal stem cells and their secretome partially restore nerve and urethral function in a dual muscle and nerve injury stress urinary incontinence model. Am J Physiol Renal Physiol 308:F92-F100.

39. Hidas G, HJ Lee, A Bahoric, MS Kelly, B Watts, Z Liu, S Saharti, A Lusch, A Alamsahebpour, et al. (2015). Aerosol transfer of bladder urothelial and smooth muscle cells onto demucosalized colonic segments for bladder augmentation: in vivo, long term, and functional pilot study. J Pediatr Urol 5:260e1-6.

40. Oberpenning F, J Meng, JJ Yoo and A Atala. (1999). De novo reconstitution of a functional mammalian urinary bladder by tissue engineering. Nat Biotechnol 17:149-155.

41. Raghavan AM and PJ Shenot. (2009). Bladder augmentation using an autologous neo-bladder construct. Kidney Int 76:236.

42. Baskin LS, SW Hayward, P Young and GR Cunha. (1996). Role of mesenchymal-epithelial interactions in normal bladder development. J Urol 156:1820-1827.

43. Jiang HH, HQ Pan, MA Gustilo-Ashby, B Gill, J Glaab, P Zaszczurynski and M Damaser. (2009). Dual simulated childbirth injuries result in slowed recovery of pudendal nerve and urethral function. Neurourol Urodyn 28:229-235.

44. Kajbafzadeh AM, A Elmi, SS Talab, SA Esfahani and A Tourchi. (2010). Functional external anal sphincter reconstruction for treatment of anal incontinence using muscle progenitor cell auto grafting. Dis Colon Rectum 53:1415-1421.

45. Yiou R, JY Hogrel, CM Loche, FJ Authier, P Lecorvoisier, P Jouany, F Roudot-Thoraval and JP Lefaucheur. (2013). Periurethral skeletal myofibre implantation in patients with urinary incontinence and intrinsic sphincter deficiency: a phase I clinical trial. BJU Int 111:1105-1116.

46. LaPallo BK, JR Wolpaw, XY Chen and JS Carp. (2014). Long-term recording of external urethral sphincter EMG activity in unanesthetized, unrestrained rats. Am J Physiol Renal Physiol 307:F485-F497.

47. Jack GS, FG Almeida, R Zhang, ZC Alfonso, PA Zuk and LV Rodriguez. (2005). Processed lipoaspirate cells for tissue engineering of the lower urinary tract: implications for the treatment of stress urinary incontinence and bladder reconstruction. J Urol 174:2041-2045.

Address correspondence to: Yan Wen, $M D$

Department of Obstetrics/Gynecology, G323 Stanford University School of Medicine Stanford, CA 94305-5317

E-mail: yanwen@stanford.edu

Received for publication November 2, 2015 Accepted after revision January 16, 2016

Prepublished on Liebert Instant Online January 20, 2016 